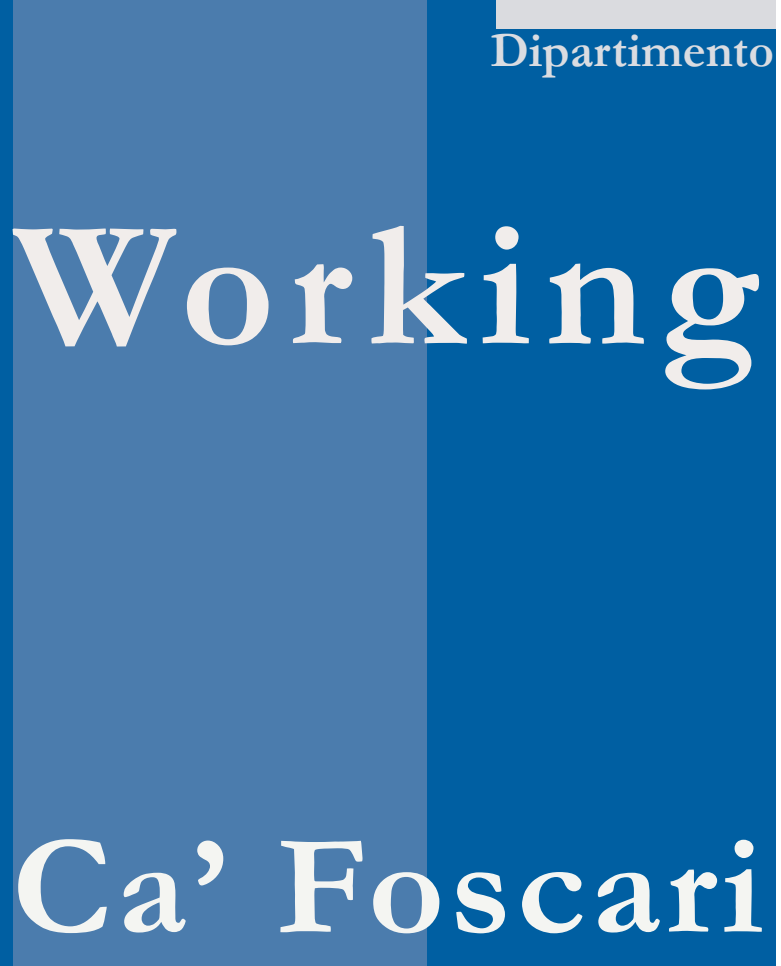

Scienze Economiche

Paper

Department

of Economics

University of

Venice

Massimiliano Caporin

Domenico Sartore

Methodological Aspect of Time Series

Back-Calculation 


\title{
Methodological Aspects of Time Series Back-Calculation
}

\author{
Massimiliano Caporin \\ University of University of Padua
}

Domenico Sartore

University of Venice and SSAV

First Draft: December 2006

\begin{abstract}
This paper provides the theoretical and operational framework for estimating past values of relevant time series starting from a (limited) information set. We consider a general approach that includes as special cases time series aggregation and temporal and/or spatial disaggregation problems. Furthermore, we explore the relevant problems and the possible solutions associated with a retropolation exercise, evidencing that linear models could be the preferred representation for the production of the needed data. The methodology is designed with a focus on economic time series but it could be considered even for other statistical areas. An empirical example is presented: we analyze the back-calculation of Eu15 Industrial Production Index comparing our approach with the Eurostat official one.
\end{abstract}

\section{Keywords}

benchmarking,retropolation, historical reconstruction, back-forecasting, missing past values, aggregation, disaggregation

\section{JEL Codes}

C10, C82, C50

Address for correspondence:

Domenico Sartore

Department of Economics

Ca' Foscari University of Venice Cannaregio 873, Fondamenta S.Giobbe

30121 Venezia - Italy

Phone: (++39) 0412349186

Fax: (++39) 0412349176

e-mail: sartore@unive.it

This Working Paper is published under the auspices of the Department of Economics of the Ca' Foscari University of Venice. Opinions expressed herein are those of the authors and not those of the Department. The Working Paper series is designed to divulge preliminary or incomplete work, circulated to favour discussion and comments. Citation of this paper should consider its provisional character.

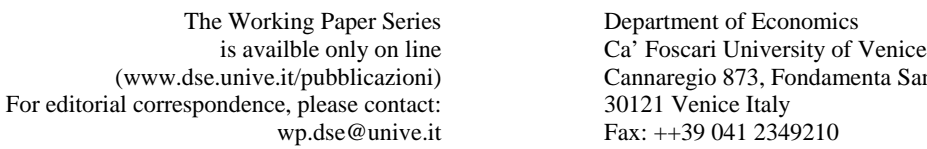




\section{Introduction}

Policy makers and practitioners often requires long time series for model evaluation, policy analysis and macroeconomic studies. However, long time series are not necessarily available at the desired frequency, with the needed spatial or sectorial coverage or they are not available at all. This unavailability may depend on various motivations: the needed indicators have been subjected to an extraordinary revision process and past values have not been reconstructed; we are searching for indicators referring to new subjects (such as the Euro Area or the enlarged European Union); production standards have been modified during last year increasing the series frequency and we need a longer series at the higher frequency. Nevertheless, time series users may require an estimate of the needed variables for past years. The aim of this paper is twofold. On the one side, it defines the back-calculation as a process for estimating past values of a time series. Furthermore, it highlights the back-calculation connections with time series temporal disaggregation, time series aggregation, the construction of proxy variables. On the other side, this paper provides a general methodological scheme that includes the available approaches and a possible technical solutions for time series back-calculation. In this last case, we point out the dependence of the back-calculation optimal design on the available information set and on the characteristics of the required and available series.

The need for having a clear definition and of rationalizing the approach derives from our practical experience with macroeconomic series; the discussion will be influenced by that and thus we will mainly refer to economic time series examples. However, the methodological treatment is general enough to be used in any statistical time series framework, including biostatistics, climatology and social sciences.

The paper has the following structure: section 2 defines the back-calculation framework while section 3 introduces our methodological scheme for the back-calculation process. Section 4 considers several statistical aspects of time series back-calculation, section 5 presents an empirical example and section 6 concludes.

\section{Defining time series back-calculation}

We first introduce some notation. Assume that our primary interest is for the time series $x_{t}$ which is available for $t=0,1, \ldots T$. Our objective is the estimation of $x_{t}$ for $t \in$ $\{-M,-M+1,-M+2, \ldots-2,-1\}$ using the information set $Z=\left\{x_{t}, Y_{t}, K_{i(t)}, W_{j(t)}\right\}$ where: for the set $Z, t=-M,-M+1, \ldots T$ and all variables can have initial and/or final missing values (as an example a related series could be available from period $j$ to period $i$, with $-M<j<0$ and $0<i<T$ - internal missing values may require specific solutions which are not considered in this paper); $Y_{t}$ is a set of variables with the same frequency as $x_{t}$ (i.e. if $x_{t}$ is a quarterly series $Y_{t}$ contains quarterly variables); $K_{i(t)}$ is a set containing series observed at a frequency lower than $x_{t}$ (i.e. if $x_{t}$ is a quarterly series $K_{i(t)}$ contains annual figures) and $i(t)=a+b t$ (in the quarterly-annual case with annual series observed at the end of the fourth quarter and $-M$ is the first quarter of an year, $a=0$ and $b=4) ; W_{j(t)}$ is a set containing series observed at a frequency higher 
than $x_{t}$ (i.e. if $x_{t}$ is a quarterly series $W_{j(t)}$ contains monthly figures) and $j(t)=c+d t$ (in the quarterly-monthly case with the quarterly series observed the third, sixth, ninth and twelfth month of the year and $W_{j(t)}$ starting the first month of an year, $c=0$ and $d=1 / 3)$.

Definition 1 A time series "back-calculation" is the estimation or approximation of $x_{t}$ for $t \in\{-M,-M+1,-M+2, \ldots-2,-1\}$ when the following conditions are jointly satisfied:

i) the set $Y_{t}$ does not contain a complete and exhaustive spatial or sectorial disaggregation of $x_{t}$ for $t \in\{-M,-M+1,-M+2, \ldots-2,-1\}$ (i.e. if $x_{t}$ is the industrial production index, $Y_{t}$ does not contain the sectorial industrial production indices for the needed time span or if $x_{t}$ is the Gross National Product, $Y_{t}$ does not contain the regional gross product);

ii) the set $K_{i(t)}$ does not contain a complete and exhaustive temporal aggregation of $x_{t}$ (i.e. if $x_{t}$ is the quarterly Gross National Product, $Y_{t}$ does not contain the annual Gross National Product);

iii) the set $W_{j(t)}$ does not contain a complete and exhaustive temporal disaggregation of $x_{t}$ (i.e. if $x_{t}$ is the quarterly industrial production index, $Y_{t}$ does not contain the monthly industrial production index);

The consequences of a violation of the previous conditions and of their effects on the backcalculation, will be discussed in a later section when dealing with model choice. Note that point i) may seem to be incomplete since it deals only with contemporaneous disaggregation, and not with contemporaneous aggregation. In fact, the availability within the set $Z$ of an aggregated variable which contains $x_{t}$, does not necessarily provide optimal information. The patterns of this aggregate variable are influenced by the dynamic and the properties of the other components. As an example, consider the Industrial Production Index on the one digit NACE Rev. 1 classification: it contains the main economic sectors, A agriculture and fishing, C mining, D manufacture and so on. If our purpose is the back-calculation of IPI for the A sector and we have the Total IPI, we cannot safely use it as our primary information source. In fact, its behavior is highly dependent on the Manufacture sector. In that case alternative data sources should be considered.

The term "back-calculation" was chosen to identify the described problems because it is used within EUROSTAT, and it identifies the methodological and empirical issues related to the estimation of unavailable past values of relevant economic variables.

\section{Back-calculation methodology: a step-by-step guide}

Up to this point we have just defined the environment. However, the main interest of practitioners refers to specific back-calculation problems and some questions may naturally arise: how we chose the content of $x_{t}$, what does $K_{i(t)}, W_{j(t)}$ and $Y_{t}$ contain; how can we estimate the past values of $x_{t}$. This section focuses on the four steps that in our view 
must be considered in order to solve a back-calculation problem. The following section will instead discuss the technical and statistical aspects of the estimation of past values for the series of interest.

\section{Step 1: Planning}

Before considering the technical aspects of the back-calculation problem, some points must be clarified. First of all, we must define $x_{t}$ from a statistical production point of view: we have to specify if we are interested in, say, the unemployment compiled with the International Labor Office standards or according to a national classification standard; alternatively, we should define if want to back-calculate the Harmonized Index of Consumer Prices using EUROSTAT standards or according to the national definition; we must also specify if we are interested in the raw series or in the seasonally adjusted one.

Furthermore, we should define $M$, the back-calculation "optimal" horizon. Two cases may be considered: $M$ is fixed a-priori given some specific needs of policy makers or users of the back-calculated $x_{t}$ series; alternatively we could consider a data-driven specification of $M$, that is letting the researcher to fix a reasonable value of $M$ on the basis of the available data. Moreover, whenever $M$ is fixed a priori and is large, there is no reason to assume that the available information will allow a complete back-calculation (i.e. we maybe not able to provide a series starting in $M$ ). A discussion on the data-driven definition of the back-calculation horizon is included in the following section.

\section{Step 2: Data availability}

Once we defined $x_{t}$, the second step focuses on the information set $Z=\left\{x_{t}, Y_{t}, K_{i(t)}, W_{j(t)}\right\}$. The object of this phase is to collect all the available information related to $x_{t}$. Within this step, several aspects must be considered:

i) the sources: we have to search for data at National Statistical Institutes, National Banks, international organizations (European Central Bank, OECD, UN...), data providers (Datastream, Reuters...) and in general at all institutions which may provide some information. The search must not be limited to the time series figures but should also collect the production methodologies (including information on the series definition, the possible adjustments for seasonality, working days, outliers...);

ii) $x_{t}$ series dimension: we have to search for temporal, spatial and sectorial aggregated or disaggregated figures of $x_{t}$ as well as for series measuring the same quantities but on different definitions; in this last case, we include both different standards (such as for the unemployment case) as well as different data adjustments (such as for seasonality, outliers, or working days);

iii) series related to $x_{t}$ : we have to search for proxies of $x_{t}$ which could be used when the analysis of $x_{t}$ series dimension produced poor results. These series may belong to $Y_{t}, K_{i(t)}$ or $W_{j(t)}$ and may measure a larger or smaller geographical area, one of the components of $x_{t}$ or they can be referred to series containing $x_{t}$; furthermore, these related series may be suggested by a (economic) theory that postulate a relation between the searched indicator and $x_{t}$. 
This point takes into consideration the data reliability which is generally attached to the source. National statistical institutes and international organizations are generally reliable sources, while companies that distribute data on the internet without specifying their source are generally more unreliable. In this last case, data errors could be more frequent. Obviously, unreliable data and sources should be carefully used and considered.

We stress that this step is a fundamental one. A proposal on the possibility of the back-calculation and on the possible back-calculation range can be rigorously structured only after a careful examination of the available data. In this step, the support coming statistical institutes will be fundamental, in particular for providing the largest possible access to the available data, even unofficial or referring to old production standards. Furthermore, information regarding possible alternative data sources available internally at national statistical institutes should be considered for improving and enlarging the back-calculation information set.

\section{Step 3: Strategy}

Once we have collected all the available information and series we can proceed to a comparative analysis whose final purpose is the strategy definition. That is, we must decide which statistical estimation approach to use. Furthermore, only at this stage we have all the information necessary to specify if we are dealing with a back-calculation problem. Alternative preferred approaches may include aggregation or time series disaggregation problems. This step is the core of the methodology and will be analyzed in the following section. The choice of the estimation approach strictly depends on the available data, in particular on their coverage and on their statistical quality. Moreover, this phase may provide a data-driven choice of $M$, the back-calculation horizon. Finally, the strategy can include a preliminary data analysis, a sensitivity analysis which may be useful for model choice and additional tests which could be of help for the definition of an optimal back-calculation.

\section{Step 4: Production and updates}

We collected the data and we specified the estimation strategy, next step is straightforward: apply the estimation approach to the information set and get the back-calculated series. However, this does not necessarily correspond to the final activity. In fact, official statistics are often revised requiring a continuous update of the back-calculation production. Assume that we back-calculated a Euro Area aggregate using a set of national series. Furthermore, assume that later some National Statistical Institute implemented themselves a back-calculation extending their data availability. In that case the whole process should be reconsidered, updating the new information and taking it into consideration within the strategy step.

Furthermore, if our primary interest is on series referred to an aggregate of countries, we assume that the National Statistical Institute back-calculations are more reliable than the possible retropolation produced by an independent researcher. In fact, the NSI have access to the original data and their information set is larger than the one available to non internal users. For this reason, an update of national series depending of a backcalculation implemented by NSI should be carefully considered by an independent re- 
searcher. Clearly, before deciding to revise a back-calculation strategy for an aggregate (as an example for Euro Area), we must consider the relevance of the revised data with respect to the aggregate to be measured. In that case, a threshold could be used. We could require a revision of the back-calculated series when at least, say, the $10 \%$ of the relevant aggregate (using the appropriate weights over the aggregate) has changed, or at least, say, 5 countries have provided a revised back-calculation, or at least one of the major, say, five countries over the aggregate, has modified its national series.

\section{A data driven back-calculation approach}

The definition of the estimation approach is a fundamental step in any statistical problem. In the back-calculation case, this step is mostly based on the available data. Furthermore, the data themselves define if we are considering a back-calculation problem or something different. In the following paragraphs we will consider several aspects related to the back-calculation strategy which jointly considered allow a correct model definition.

\subsection{Aggregation and disaggregation}

Only once the data have been collected we can define if we are in a back-calculation problem or if the available information allow the use of alternative approaches available in the literature. Using the conditions of Definition 1 we can state the following:

a) whenever $Z$ contains a complete disaggregation of $x_{t}$ for $t \in\{-M,-M+1,-M+$ $2, \ldots-2,-1\}$ (violating condition i) we are facing an aggregation problem (spatial or sectorial);

b) whenever $Z$ contains a complete temporal aggregation of $x_{t}$ for $t \in\{-M,-M+1$, $-M+2, \ldots-2,-1\}$ (violating definition ii) we are facing a temporal disaggregation problem;

c) whenever $Z$ contains a complete temporal disaggregation of $x_{t}$ for $t \in\{-M,-M+1$, $-M+2, \ldots-2,-1\}$ (violating definition iii) we are facing a temporal aggregation problem.

Case a) is considered by Gonzalez Minguez (1997) and Beyer, Doornick and Hendry (2000 and 2001) in the construction of aggregated series for the Euro Area, dealing in particular with the exchange rate problem. This case can be generalized including the aggregation of sectorial series in the estimation of total figures. Case c) is similar to case a) the only difference is in the dimension of the involved series which is here in the time domain only and does not consider sectorial or spatial dimensions. Finally, case b) is the most interesting. Temporal Disaggregation problems have been considered since the seminal work of Chow and Lin $(1971,1976)$, recently extended by Fernandez (1981), Litterman (1983), Santos Silva and Cardoso (2001), Di Fonzo (2003a and 2003c) and therein cited references. In general we can distinguish two subcases: 
$\left.\mathrm{b}_{1}\right) Z=\left\{x_{t}, K_{i(t)}\right\}$ - that is, there is no information available at the $x_{t}$ frequency but only at a lower frequency. The following strategies can be considered: i) to use a purely statistical temporal disaggregation approach, such as the Denton moving preservation principle (Denton, 1971); 2) to use a proportional distribution estimating weights with the available $x_{t}$ observations; 3 ) to extract with a structural or linear model the components of $x_{t}$, project them into the past and then use a Chow-Lin approach;

$\left.\mathrm{b}_{2}\right) Z=\left\{x_{t}, Y_{t}, K_{i(t)}\right\}$ - that is, there is something more than in case $\left.\mathrm{b}_{1}\right)$, there exist some information on series related to $x_{t}$. This setup is generally named constrained retropolation and is partially discussed in Di Fonzo (2003b). The related series included in $Y_{t}$ could be used to back-calculate $x_{t}$, in order to obtain a preliminary estimate. However, we can also use the information included on a lower frequency series, that can be used for benchmarking the back-calculated series. The preliminary estimate of $x_{t}$ past values can be used as the best related indicator in a constrained retropolation framework. We refer to the combined used of back-calculation and other methods (aggregation and disaggregation) as "mixed approaches". The availability of an aggregated estimate or in general of additional information at different time frequencies can be considered as a plus with respect to the standard back-calculation problem. The derivation of a preliminary backcalculated series is strictly related to the construction of a proxy variable: in fact, the use for the estimation of a desired series of related series with different coverage and frequency or suggested by some theoretical linkage is the standard problem of constructing a proxy variable.

\subsection{Back-calculation}

Assume that Definition 1 is satisfied; we are then considering a back-calculation problem. However, several cases may realize:

a) $Z=\left\{x_{t}\right\}$ - there is nothing more than the series we are considering;

b) $Z=\left\{x_{t}, Y_{t}\right\}$ - there are related series without missing values;

c) $Z=\left\{x_{t}, Y_{t}\right\}$ - there are related series with missing values.

In case a) the only possibility is the use of an ARIMA approach. Two solutions are available: estimate an ARIMA on the current series, reverse the model and use it to produce some forecasts; reverse the series, fit an ARIMA model and use it to produce forecasts. Both approaches presents some problems. Focus at first on time series temporal reversion: this approach must be carefully considered. The econometric literature includes a time reversibility test, due to Ramsey and Rothman (1996). However, this test requires the symmetry of the series (i.e. no trends, no asymmetric seasonal components and no asymmetric cycles). Unfortunately, it is know that economic time series which are influenced by the business cycle are asymmetric, see Sichel (1993), Clements and Krolzig (2003) and therein cited references. In addition, reversing the time path of a series does not necessarily have a clear economic interpretation, despite from a statistical point of view it could be strongly supported. As a result, we believe that time series reversion should not be considered for the back-calculation of economic time series. 
Alternatively, under an ARIMA approach, forecasts can be made only for a limited number of steps into the past. Otherwise, either we will estimate only a tendency, or the forecasts will converge to their long run level, or they will explode. Differently, the reversion of MA terms is useless since past values of the innovation term are not available. In that case a stochastic simulation approach could be considered: we could use a bootstrap sampling from the estimated residuals of the fitted MA model. However, the reliability of the resulting series could be questionable.

We suggest to estimate the back-calculations of cases b) and c) within a regression framework. We propose to specify for the back-calculation a linear regression model over $\mathrm{m}$-th order differenced series, possibly extended with ARMA terms in the residuals. The general model we propose is the following

$$
\Delta^{m} x_{t}=\beta \Delta^{m} Y_{t}+\delta D_{t}+\gamma T_{t}+\Phi^{-1}(L) \Theta(L) \varepsilon_{t}
$$

where $Y_{t}$ contains the related indicators possibly including the constant, $D_{t}$ is a set of seasonal dummies (if needed), $T_{t}$ is the time trend and $\varepsilon_{t}$ is an innovation process. The model can be estimated by maximum likelihood and standard tests can be used to evaluate the estimated coefficients and the residuals. Clearly, we can estimate the regression model only if the relevant series and the related indicators are available on an (even limited) overlapping sample (in case this sample is very small, the back-calculation could provide unreliable past observations). Once the coefficients have been estimated, the back-calculated differenced series is obtained as follows

$$
\widehat{\Delta^{m} x_{t}}=\hat{\beta} \Delta^{m} Y_{t}+\hat{\delta} D_{t}+\hat{\gamma} T_{t} \quad t<0
$$

Then, the back-calculation of the levels are given by a recursive equation. Under the case $m=1$, we can back-calculated levels using the following equations

$$
\begin{aligned}
\hat{x}_{-1} & =x_{0}+\widehat{\Delta x_{0}} \\
\hat{x}_{-l} & =\hat{x}_{-l+1}+\Delta \widehat{x}-l+1 \quad l=2,3, \ldots, M
\end{aligned}
$$

Even if the simple linear regression model of equation (1) is static, some dynamic is included if $m \neq 0$. Note that the ARMA terms included in the residuals are not used in the estimation of the back-calculated m-th order differences $\Delta^{m} x_{t}$ and of the level values. They are included for increasing the efficiency of the mean parameters and for allowing a finer regression selection procedure that may take place over the set of indicators and dummy variables considered.

Case b) directly fits with equation (1) while case c) requires a preliminary step. Remember that under this hypothesis, the information set contains related series with initial or final missing values, i.e. the various related series have a different time and/or spatial and/or sectorial coverage (as stated before, the case of internal missing values is not considered here). An example may clarify the situation: assume that we are interested in estimation past values for the EU15 GDP from 1980. Furthermore, the information set contains the past GDP values for 10 out of the 15 countries with 6 series starting in 1980 while the remaining start in 1985 . We can then back-calculate $x_{t}$ with several alternative approaches. In fact, we can use only the series available for the whole back-calculation 
sample, directly including them in $Y_{t}$. Alternatively, we can extract a proxy from the available information and then we can use equation (1). Finally, we can also proceed with several back-calculation steps progressively extending the back-calculation horizon. We suggest to consider whenever possible this last estimation approach.

We motivate our preference referring again to the GDP example. Assume now that the available EU15 GDP series starts in 1990 and that we have the following available data: Germany, France, Spain, Italy and UK GDP series starting in 1980; Belgium, The Netherlands and Finland GDP series from 1985 (these coverage do not correspond to the reality - the problems referred to the GDP prices, its base year, the seasonal adjustment and the system of account are not considered for the sake of exposition). Our purpose is the back-calculation of EU15 GDP back to 1980. We have two groups of variables available with different coverage. If we do not consider Belgium, Finland and The Netherlands series and use only the six countries available for the whole backcalculation sample we get back to case b). However, using this approach the backcalculation procedure will turn out to be simpler, but not all the available information will be used. All the available data will instead be used if we consider a set of backcalculations, that progressively extend the available range. We can thus split the backcalculation into two parts: at first, back-calculate Euro 15 GDP back to 1985 using all the available information, and then back-calculate the Euro 15 from 1980 to 1984 using a different (and smaller) information set. In this second approach, we use all the available data and the back-calculation turns out to be more efficient. As a general rule, we suggest the use of this approach given that it can be designed in order to use all the available indicators and series even if they have a different time coverage.

We consider again the example of the GDP back-calculation in order to highlight a specific aspect of our preferred back-calculation approach. Assume we build two indicators: a Euro 5 series which is available from 1980 and a Euro 7 series which starts in 1985 (for the sake of exposition we refer to the indicators and not to the inclusion of the two variable sets on the regression equation). In principle, we can follow two approaches: we can back-calculate Euro 15 on Euro 7 back to 1985 obtaining a new Euro 15 series and then, in a second step, back-calculate the newly available Euro 15 series back to 1980 using the Euro 5 series (case A); alternatively, we can back-calculate the Euro 7 series back to 1980 using the Euro 5 series and then in the second step, back-calculate the Euro 15 series back to 1980 using the newly available Euro 7 series (case B). In both cases we will make use of the whole information set. 


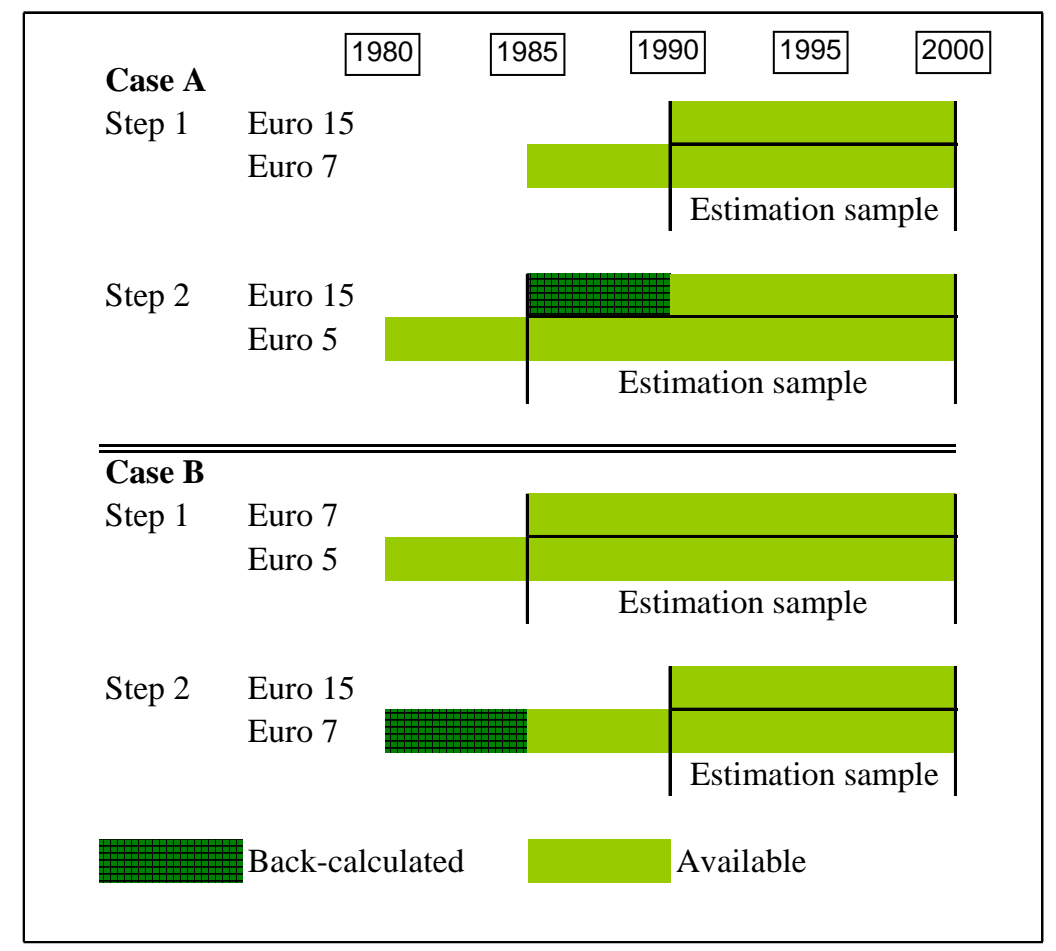

Figure 1: alternative back-calculation approaches

The differences among the two approaches are highlighted by Figure 1. Note that in the second step, Case A involves a regression based on estimated values while case B uses only already available indicators. The two approaches will provide close results. However,Case B avoids the inclusion of estimation errors on the data used in the second step. Furthermore, we believe that Euro 5 is by construction the preferred indicator in the retropolation of Euro 7, while Euro 7 is a preferred indicator for the retropolation of Euro 15. This is not the case when we use Euro 5 in estimating Euro 15. In fact, Euro 7 contains more information than Euro 5.

We close this section with some comments referred to the definition of the back-calculation optimal horizon. We have already argued that a back-calculation range a priori fixed may not be optimal. In fact, there are no reasons for ensuring that it will be covered. Using the available data, the proposed range may not be achieved for a lack of indicators. In that case, the only way to recover the past values of a series is by constructing a proxy with an adequate modeling strategy. We will not discuss this point further in the current paper.

The available data are the primary source for information about the back-calculation process. They should be considered also for the definition of the range, which could be fixed at the time of the oldest data available in the information set $Z$. This choice should be the preferred one, however it may not be optimal in some cases. Consider the back-calculation of an Euro Area aggregate back in time by using national time series of the same variable. Assume also that most countries are available from 1980 with the exception of Ireland which is available from 1975. In that case the estimation of the Euro Area aggregated in the range 1975-1979 could be based only on the information included in the Ireland series. Despite the technical possibility of producing in any case the back-calculation, the resulting series will be based on a limited and poor information 
set. This fact will affect the reliability of the produced data. Deciding where the backcalculation range should be fixed is a complex task. In fact it depends on the available data, on their reliability, and possibly on the coverage with respect to the aggregate we are interested in. Furthermore, it depends also on the estimated model, on its diagnostic and on its explanatory power. The optimal range will thus be fixed on a case by case basis after a preliminary data analysis and also benefiting from preliminary and exploratory estimations. For these reasons, we believe that there are no general rules to be followed.

\subsection{Additional aspects}

In order to perform a back-calculation, statistical properties of the series involved in the back-calculation should be analyzed. In fact, we are interested in the evaluation of the reliability of the indicators and on their usefulness in the estimation backward of the series of interest. Assuming we are using a single indicator, it will be a relevant indicator if it share a common trend with the series to be back-calculated. In this step a cointegration analysis could be useful. However, we evidence that if an error correction representation exists, the error correction term cannot be used in the back-calculation. The motivation is the same we reported when analyzing dynamic models.

Before proceeding to the back-calculation we must also decide if we will work on the levels, the logs, or on any difference of the series. The choice must be based on the purposes of the back-calculation and on the series characteristics. If series are not integrated of any order, the levels provide a good back-calculation of the tendency of the series, while the difference or the log-returns provide a good back-calculation of the growth rates. If series have a seasonal pattern, seasonal (log-)differences can be considered in order to back-calculate yearly growth rates if series are integrated at the seasonal level. Again, there is not a preferred solution: all methodologies could be used, and compared.

In principle, we could choose one of these cases to perform the analysis, alternatively, we can compute all the back.calculations and then consider a combined back-calculation approach. In this case we can refer to the literature of combined forecast, see Clemens (1989) and Granger (1989) among others. The combination of different approaches allows a reduction of the 'model risk': we are not aware of which is the correct model, therefore combining a set of possible alternative solutions we consider all the available information in the most efficient way.

A further point that emerges when having different alternative back-calculated series refers to the choice among one of them or on a combination of them. In sample sensitivity analysis can be used to choose model specification and possible forecast combination. In this case, RMSE, AMSE and Theil U index could be used as measures of back-calculation accuracy. 


\section{A case study: retropolation of EU15 Industrial Production Index}

In order to provide an empirical example of our back-calculation approach we consider the estimation of the monthly EU15 Industrial Production Index. We refer to the index for Total Industry excluding construction (NACE Rev. 1.1 sectors C, D and E - mining and quarrying, manufacture and energy), seasonally unadjusted, working day adjusted, in 2000 base year. The EU15 and national series are available on the NewCronos database (EUROSTAT database, last access September 2005) with the following coverage and national weights over the total EU15:

Table 1: IPI Total Industry excluding construction (WDA - 2000 base year)

\begin{tabular}{|l|l|l|l|l|l|}
\hline Country & First Obs. & Weight & Country & First Obs. & Weight \\
\hline Austria & January 1996 & 2.5 & Italy & January 1990 & 13.9 \\
\hline Belgium & January 1970 & 3.2 & Luxembourg & January 1970 & 0.2 \\
\hline Denmark & January 1985 & 1.9 & Portugal & January 1990 & 1.3 \\
\hline Finland & January 1990 & 2.0 & Spain & January 1980 & 6.9 \\
\hline France & January 1990 & 14.2 & Sweden & January 1990 & 3.2 \\
\hline Germany & January 1978 & 26.4 & The Netherlands & January 1970 & 4.1 \\
\hline Greece & January 1995 & 0.8 & United Kingdom & January 1986 & 17.5 \\
\hline Ireland & January 1980 & 2.1 & EU15 & January 1986 & 100.0 \\
\hline
\end{tabular}

Actual national and EU15 coverage as reported in NewCronos (first available observation - availability at the end of August 2004) - country weight on total EU15

We compare our strategy with the EUROSTAT official one which is based on the same information set. Nowadays, EUROSTAT adopt a data-driven definition of $M$ linking it to the availability of at least the $60 \%$ of the EU15. EUROSTAT measures the threshold on the basis of country weights over the EU15 on the current basis year. Given $M$, EUROSTAT computes the EU15 series by a weighted average of national indices using weights reported in Table 1. Whenever, one of more countries are missing, lower geographical coverage level indices are determined (that is, if one country is missing for dates before January 1996, a weighted EU14 series is computed). Then the drift and level shift of these proxies are matched to the available total coverage index (see http://europa.eu.int/newcronos/suite/info/ notmeth/en/theme4/ebt/ebt.htm?action= notmeth\#updss for a description of the process). For the previous reasons, the EU15 series is available from January 1986 when United Kingdom series starts. Furthermore, the EU15 series is exactly EU15 series only from January 1996, when Austria series starts. Practically, EUROSTAT is not properly considering the data availability step and is not making any estimation, but simply some adjustments. This strategy produces several inconsistencies in the official EU15 series before 1996. In fact, before that date, trend and seasonal patterns are referred to a different geographical area. As a result, the EU15 series is not comparable through time since after 1996 is an EU15 computed on total coverage while before 1990 is an EU15 series computed (and not estimated) on 7 countries. The inconsistency will be evident in a few steps. 
The following exercise is simply an example of our approach and should not be considered as the best solution for back-calculating EU15 IPI. In fact, by using data available from the OECD and National Statistical Institutes, and data available in NewCronos on the NACE classification with 4 digit precision, a different and more efficient strategy can be designed. This approach requires a longer discussion, a deeper data analysis and an elevate number of estimates and comparisons. Given that the main contribution of this paper is in the presentation of the methodology, we prefer to focus on a simpler example whose purpose is not the update of official data. Such different topic is under development at EUROSTAT following the methodology presented in this paper and using the largest information set available.

In this exercise, we assume that the only information is given by the NewCronos national series. Moreover, we stress that this is exactly the same dataset actually used by EUROSTAT for the back-calculation of the IPI series. Given the available data, we plan to back-calculate the EU15 series from 1980. A further extension could be considered but it would be based on a very limited information set that could excessively bias the results (the coverage is of the $31.9 \%$ from 1978 to 1980 and $6.3 \%$ from 1977 back to 1970). In order to evidence the properties and the reliability of our approach, we assume that the actually available EU15 official series starts in January 1996, when all national series are available (note that we will not use in our regression estimation the actual official EU15 series which starts in 1986).

By using the weights of Table 1 we compute the following related indicators (they correspond to a partial measure of the needed index, or, to a measure on a different geographical area): EU6 starting in 1980, EU7 starting in 1985, EU8 available from January 1986, EU13 from January 1990 and EU14 from January 1995.

The back-calculated growth rates were obtained with the following set of regressions: EU6 is used to retropolate EU7 series; the estimated EU7 series is then used as a related indicator for the EU8 series; EU13 series back-calculation uses the estimated EU8 series; EU14 series retropolation uses the estimated EU13 series; and, finally, EU15 series reconstruction is based on the estimated EU14 series. All estimated models include a set of dummies and ARMA terms on the residuals. With the retropolated growth rates we computed an estimate of the EU15 series levels. Figure 3 reports a the back-calculated EU15 series. Furthermore, Figure 4 reports on the range from January 1986 to December 1995, the discrepancies between the official series and the retropolated one computed both on the levels and on the growth rates. Figures 5 finally eports the annual growth rates of the official and back-calculated series and the differences between the two. 


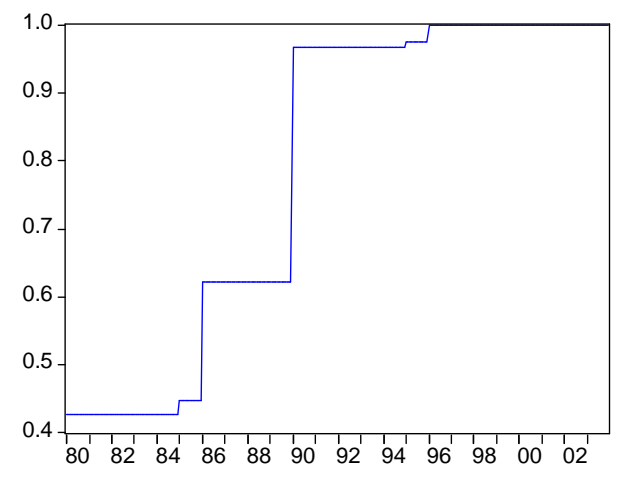

Figure 2: IPI coverage over Euro15

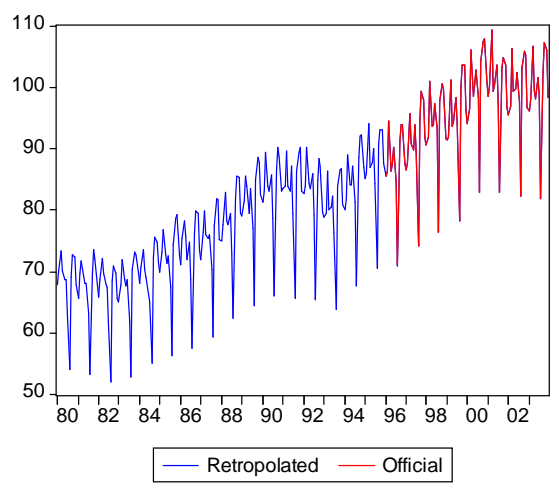

Figure 3: retropolated Euro15 series
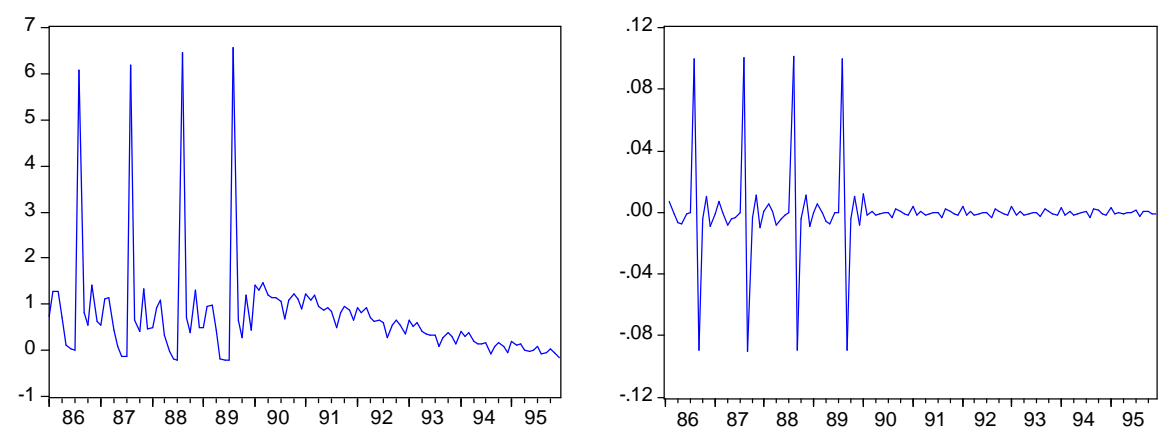

Figure 4: discrepancies between official and retropolated series over the range 1986-1995

- levels (left) and log-differences (right)
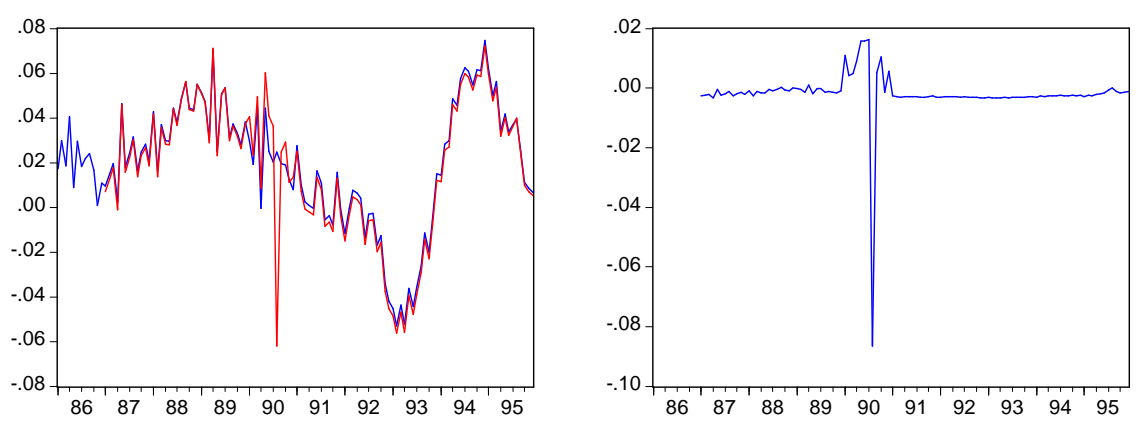

Figure 5: comparing annual log-differences over the range 1986-1995: retropolated (blue) - official (red) (left) and discrepancies (right)

It is evident that the discrepancies have a seasonal component in the range 1986-1989; less evidently, a trend is present in the whole range, as well as a seasonal effect in the second part of the back-calculated range. The differences are due to the production process adopted by EUROSTAT: a trend and shift adjustment made on the EU8 series to chain it to the available EU13 series is not equivalent to the estimation of EU13 (and then EU15) using EU8 and a set of deterministic and stochastic components. In fact, the correction used by EUROSTAT does not recover the seasonal component of EU15 which is 
evidently different from the one of EU8 given the missing data of 5 countries (France and Italy included) whose weight on EU15 is about the $34 \%$. Similarly, the trend of the series is affected. As a result, the EU15 IPI series actually available is internally inconsistent and present discrepancies with respect to the true unavailable series both on the trend and on the seasonal components. Comparing the annual logarithmic differences one can note that the cyclical seems not to be affected, apart the inclusion of an outlier. We stress that this huge discrepancy is not a true outlier but strictly depends on the actual back-calculation process adopted by EUROSTAT. With a simple back-calculation strategy, which is not the most efficient since we used only a limited information se,t we evidence a serious problem of the actual official EU15 series of Industrial Production: seasonal pattern is not consistent and includes at least one strong structural break; furthermore, there is a limited deviation in the trend. Finally, we evidence that using the same dataset used by EUROSTAT our methodology could increase the back-calculation range and improve the internal consistency of the produced series. However, this is not the optimal solution but a simple example given that only a limited information set has been used.

\section{Concluding remarks}

This paper presents a methodological approach for back-calculation problems, that is for the estimation of past values of relevant series by using a limited information set. We consider a general framework that includes a set of possible cases ranging from the temporal and/or spatial aggregation, the temporal and/or spatial disaggregation, the retropolation and constrained retropolation. We provide a scheme to be used for backcalculation problems and an empirical example showing the advantages of our approach compared to the one actually used by EUROSTAT in the reconstruction of the EU15 Industrial Production Index. In this paper we evidence a preference for linear regression models, however, alternative approaches could be considered. The extension to different models is left for future researches.

\section{References}

Beyer A., J.A. Doornick And D.F. Hendry (2000), "Reconstructing Aggregate Euro-zone Data", Journal of Common Market Studies, 38-(4), 613-624

Beyer A., J.A. Doornick And D.F. Hendry (2001), "Constructing historical Eurozone data", The Economic Journal, 111, F102-F121

Box, G.E.P. And G.M. Jenkins (1970) Time series analysis: Forecasting and control, San Francisco: Holden-Day.

Chow G. And A.L. Lin (1971), "Best linear unbiased interpolation, distribution and extrapolation of time series by related series", The Review of Economics and Statistics, 53: $372-375$.

Chow G. AND A.L. Lin (1976), "Best linear unbiased estimation of missing observations in an economic time series", Journal of the American Statistical Association, 71: 719-721. 
Clemen R. (1989), "Combining forecasts: A review and annotated bibliography", International Journal of Forecasting, 5, 559-583

Clements, M.P. And Krolzig, H.-M. (2003). "Business Cycle Asymmetries: Characterisation and Testing based on Markov-Switching Autoregressions", Journal of Business and Economic Statistics, 21, 196 - 211

Denton F.T., (1971), "Adjustment of monthly or quarterly series to annual totals: an approach based on quadratic minimization", Journal of the American Statistical Association, 1971, 66, 99-102.

Di Fonzo, T. (2003a), "Temporal disaggregation using related series: log-transformation and dynamic extension", Rivista Internazionale di Scienze Economiche e Commerciali, 50, 3, pp. 371-400.

Di Fonzo, T. (2003b), "Constrained retropolation of high-frequency data using related series. A simple dynamic model approach", Statistical Methods \& Applications, 12, pp. 109-119.

Di Fonzo, T. (2003c), "Temporal disaggregation of economic time series: towards a dynamic extension", European Commission (Eurostat) Working Papers and Studies, Theme 1, General Statistics (pp. 41).

Fernandez R.B. (1981), "A methodological note on the estimation of time series", The Review of Economics and Statistics, 63: 471-478.

Gonzalez Minguez, J.M. (1997), "The back calculation of nominal historical series after the introduction of the European Currency (An application to the GDP)", Banco de Espana, Servicio de Estudios, Documento de Trabajo n. 9720

Granger, C.W.J. (1989), "Invited review: combining forecasts - 20 years later", Journal of Forecasting, 8, 167-173

Litterman R.B. (1983), "A random walk, Markov model for the distribution of time series", Journal of Business and Economic Statistics, 1: 169-173.

Ramsey, J.B., Rothman, P., (1996). "Time irreversibility and business cycle asymmetry", Journal of Money, Credit and Banking 28, 3-20.

Santos Silva J.M.C. And F.N. Cardoso (2001), "The Chow-Lin method using dynamic models", Economic Modelling, 18: 269-280.

Sichel, D.E., (1993), "Business Cycle Asymmetry", Economic Inquiry, 31, 224-236

Stram D.O. And W.W.S. Wei (1990), "Disaggregation of time series models", Journal of the Royal Statistical Society, 1990, 52, 453-467. 